\title{
UNA APROXIMACIÓN A LA CONSTRUCCIÓN DE IDENTIDAD DE MUJERES CADETES EN LA ESCUELA MILITAR DEL EJÉRCITO DE CHILE
}

\section{Claudia Pérez Bravo ${ }^{1}$}

\begin{abstract}
RESUMEN
El presente artículo reflexiona sobre los hallazgos de una investigación enmarcada en el ámbito de la educación superior y género. Se interesa por la incorporación de las mujeres al Ejército, y en particular por su proceso de construcción de identidad. Se analizan los discursos, prácticas y procedimientos vividos en su formación entendiendo que ellos constituyen materiales de identificación que delinean una cierta forma de ser y actuar. Se utilizó una metodología de investigación cualitativa, realizando ocho entrevistas semiestructuradas y en profundidad, a mujeres cadetes que cursan el programa educativo en el año 2010.

En la educación militar se forja un sujeto institucional al tiempo que un sujeto masculino. Agudizando la división masculino/femenino se refuerzan roles y representaciones sociales tradicionales, y se promueve la aceptación naturalizada y sexualizada de una identidad, por complemento para el caso de las mujeres.
\end{abstract}

Palabras clave: mujeres, identidad, fuerzas armadas.

\section{AN APPROACH TO THE CONSTRUCTION OF AN IDENTITY FOR CADET WOMEN IN THE CHILEAN ARMY MILITARY SCHOOL}

\begin{abstract}
This article reflects on the findings from a research framed in the field of higher education and gender. It addresses the incorporation of women into the Army, and in particular the processes of identity construction.

In doing that, it analyzes discourses, practices and experienced processes in their training as an identification material that outlines a certain way of being and acting.

Military education forges an institutional subject while a male subject. Exacerbating the male/ female roles separation, it reinforces the traditional social representation and promotes the natural and sexualized acceptance of an identity, by complement in the case of women.
\end{abstract}

Keywords: women, identity, Army.

1 Departamento de Estudios Generales. Universidad Academia de Humanismo Cristiano. Santiago, Chile.

Contacto: zzara23@hotmail.com 


\section{UNA APROXIMACIÓN A LA CONSTRUCCIÓN DE IDENTIDAD DE MUJERES CADETES EN LA ESCUELA MILITAR DEL EJÉRCITO DE CHILE}

\section{Introducción}

En las últimas décadas nuestro país ha progresado significativamente en la igualdad entre hombres y mujeres. La existencia de un nuevo marco de leyes y políticas que promueven la mayor legitimidad que ha alcanzado la igualdad de género en la sociedad, han repercutido en una mayor igualación de las capacidades y oportunidades de hombres y mujeres (PNUD, 2010).

La educación, y en particular la enseñanza superior, es uno de los ámbitos más consolidados en la igualdad de género, lo cual se refleja en el masivo acceso y participación de las mujeres en la educación terciaria. Así es como, entre los países de Latinoamérica, Chile logra equidad en la matrícula para este nivel educativo (Papadópulos y Radakovich, 2005). Junto a ello, la reciente incorporación de mujeres a las fuerzas armadas como partes de la organización, que implica la apertura de las escuelas militares, es otro indicador muy significativo, el que desde una perspectiva simbólica permite dimensionar los diversos avances en materia de igualdad de género en todos los ámbitos de la vida pública, especialmente en educación postsecundaria.

Para el sociólogo español Carlos Echeverría (1994), la inclusión de las mujeres a las escuelas de formación militar responde a cuatros dimensiones que actúan de forma conjunta, y que visibilizan transformaciones sociales estructurales: política, que propicia el reconocimiento de los derechos de la mujer y la igualdad entre los ciudadanos; laboral, que va permitiendo y aceptando la igualdad con respecto al trabajo sin discriminación; sociológica, que asume culturalmente una mayor aproximación entre los roles tradicionales que corresponden a cada sexo y una mayor equiparación de funciones y tareas; militar, que va propiciando una mayor profesionalización del 
personal y, por lo tanto, un modelo de carrera y un mayor atractivo de reclutamiento.

La experiencia de inclusión de mujeres a las fuerzas armadas en Latinoamérica, a través de las escuelas militares, se relaciona fuertemente con el retorno a gobiernos de elección democrática. El proceso de incorporación se desarrolla de manera heterogénea, tanto en referencia a las particularidades de los contextos sociopolíticos de cada país, como a las distintas ramas (terrestre, naval y aérea), escalafones y especialidades. En este último punto, la norma general es la exclusión de mujeres de las especialidades de combate directo, a excepción de Uruguay. Esta tendencia general también es observada en países como Estados Unidos y el Reino Unido donde la incorporación de mujeres tiene larga data (Lucero, 2009).

En Chile, el ingreso a cualquiera de las organizaciones militares se realiza necesariamente a través de un modelo educativo propio y exclusivo a cada una de ellas. En específico, el sistema educativo del Ejército para la carrera de oficiales contempla tres niveles de estudio. El primero de ellos marca la entrada a la carrera militar y corresponde al proceso de formación básica, donde se proporcionan las capacidades militares asociadas al mando, conducción de pequeñas unidades, sistemas de armas y administración ${ }^{2}$. El segundo nivel de formación es coextensivo al primero, y tiene como finalidad la especialización en alguna de las áreas de tácticas al interior de la organización. El proceso de formación superior corresponde al tercer nivel educacional, en él se desarrollan programas de posgrado en distintas disciplinas de la ciencia militar.

Ahora bien, la inclusión de mujeres al Ejército de Chile como parte de la fuerza, ha requerido efectuar un conjunto de reformas a nivel político y organizacional, que permitieran generar el espacio para asimilar su ingreso y promover su desarrollo. En el plano político su incorporación, bajo el principio de igualdad de oportunidades, comienza a tomar fuerza a partir del gobierno de Ricardo Lagos (20002006), específicamente bajo la administración ministerial de la cartera

2 Ver requisitos de postulación en anexo n. ${ }^{\circ} 1$ 
de Defensa de Michelle Bachelet, periodo en el cual se constituye una Política de Integración de la Mujer a las Instituciones de la Defensa ${ }^{3}$. Las bases de esta política son ${ }^{4}$ : igualdad de oportunidades entre hombres y mujeres, integración, competencia profesional, equidad, liderazgo y planificación. Desde las organizaciones castrenses, el proceso de inclusión de las mujeres a las fuerzas armadas chilenas, ha sido vivido de manera diferente por las distintas ramas, con énfasis, enfoques y tiempos cronológicos distintos.

En el presente trabajo nos centramos en el Ejército de Chile, en el cual se puede apreciar una integración escalonada en el tiempo. Desde 1974 con la apertura de la Escuela de Servicio Auxiliar Femenino (ESAFE), las mujeres están presentes en la organización. En esta primera etapa, como indica Villalobos (2007), las funciones asignadas a las mujeres no eran propiamente militares, sino de apoyo administrativo en temas financieros, de personal, secretaría y manejo de documentos. Luego en 1995, en lo que podríamos llamar una segunda etapa, ingresan mujeres a la Escuela Militar con un programa académico de dos años; a partir de 1999 se expanden sus funciones al incorporarse mujeres en las áreas de apoyo logístico de material de guerra e intendencia. Una tercera etapa comienza en 2003 cuando se produce un importante cambio al abrirse las posibilidades para las oficiales de integrar las Armas de Apoyo de Combate, de Artillería, Ingenieros y Telecomunicaciones, ampliando así los ámbitos de

3 El producto de este trabajo se plasmó en la Separata de actualización n. ${ }^{\circ} 28$ del Libro de la Defensa Nacional, publicada en marzo de 2005.

4 Sus principales objetivos son:

1. Aplicar en el sector de la Defensa Nacional los principios de igualdad de derechos entre hombres y mujeres y de asegurar el derecho de las personas a participar con igualdad de oportunidades en la vida nacional, expresados en la Constitución Política de la República y priorizados en la acción del Gobierno.

2. Establecer los principios que rigen la participación de las mujeres en las Fuerzas Armadas como parte de la Política de Defensa. Con ello, asegurar su reconocimiento al más alto nivel y con un carácter estable, entregar reglas claras, consistentes y comunes para todas las instituciones, y de carácter público que permita su seguimiento y haga necesaria la rendición de cuentas respecto a los compromisos adquiridos.

Dichos principios orientadores son: igualdad de oportunidades entre hombres y mujeres, participación integrada de hombres y mujeres en las actividades profesionales, aplicación de criterios de competencia profesional, equidad en el trato, liderazgo en la conducción del proceso de integración, incorporación permanente a la planificación institucional.

3. Potenciar la contribución de las mujeres a las organizaciones armadas, logrando contar con los mejores recursos humanos para el cumplimiento de las respectivas misiones mediante la selección de los más capaces, sin consideración de sexo. (Villalobos, 2007; p. 5). 
desempeño del personal femenino ${ }^{5}$. Actualmente pueden optar a especializaciones secundarias, de acuerdo con las necesidades institucionales cumpliendo con los requisitos necesarios, incluyendo algunas especializaciones de combate (Libro de la Defensa Nacional, separata de actualización n. ${ }^{\circ}$ 28, 2005).

Aún teniendo en consideración la importancia que tiene este conjunto de reformas en la generación de un espacio para que las mujeres participen como parte de la fuerza en el Ejército, es evidente que un proceso de integración que aspira a construir condiciones de igualdad de derechos, oportunidades y ejercicio en una organización cuya función ha sido ejercida históricamente solo por varones, lo cual ha promovido prácticas, lenguajes y códigos que la hacen institucionalmente masculina, necesita contemplar y revisar qué sucede en su dimensión cultural. La búsqueda bibliográfica reporta escasa, sino nula, evidencia acerca de los cambios y adaptaciones que subyacen al proceso. Esto abre la posibilidad y justifica realizar investigaciones que permitan a un nivel exploratorio reconocerlos, teniendo en cuenta a las mujeres como protagonistas. En este sentido, el punto de partida fue conocer el proceso de definición de identidad que inician estas nuevas cadetes, en el transcurso de la resignificación de la cultura institucional. En este entendido la pregunta general que guía este trabajo es: ¿cómo se configura la identidad de las mujeres cadetes en el sistema de educación militar del Ejército de Chile, Escuela Matriz para Oficiales?

La identidad está en el centro de nuestro estudio, precisamente porque funciona como un punto desde donde mirar procesos más amplios y objetivos, como la experiencia de inclusión. En este sentido nos permite detectar cómo se aplican los cambios de relaciones de género, traducidos en la política de integración, y la experiencia cotidiana de las mujeres en la Escuela.

Se definieron cuatro dimensiones de análisis. La primera de ellas hace referencia a las condiciones socioculturales que subyacen y se activan en la elección de la carrera. La segunda aborda los marcadores

5 Ver Línea de Carrera de Oficiales Femeninas. Pág. 35 
de identidad constitutivos de la formación militar. La tercera apunta sobre el tratamiento de las nociones de igualdad y diferencia en la experiencia cotidiana en la Escuela y cómo ello se traspasa a las posibilidades de ejercicio en la organización. Y finalmente la cuarta dimensión explora en los referentes de género que se relevan en la construcción de la "identidad femenina" en la Escuela Militar.

\section{Aclaraciones metodológicas}

Se considera necesario situar como escenario material de investigación el nivel formativo de la educación militar del Ejército de Chile, en tanto este cumple las funciones de preparación, transmisión ideológica y socialización de la doctrina, reproduciendo el orden institucionalizado, lo cual influye en la construcción de identidad (particular y genérica, corporativa y profesional) de sus partícipes y promueve los procesos de apropiación y significación de la identidad.

El artículo se sustenta en un estudio de carácter exploratoriodescriptivo respecto de la configuración de la identidad de mujeres que cursan el nivel primario de educación en la Escuela Matriz del Ejército de Chile. Entenderemos la configuración de identidad siguiendo a Giménez (1997), quien explica que es un proceso en el cual "el individuo se ve a sí mismo -y es reconocido- como 'perteneciendo' a una serie de colectivos; como 'siendo' una serie de atributos; y como 'cargando' un pasado biográfico incanjeable e irrenunciable” (p. 5). Para tal efecto, se ha utilizado una metodología de investigación cualitativa. Vieytes (2004) plantea que "la investigación cualitativa nos propone acceder a la subjetividad del otro en pos de encontrar el mundo que a través de esa indagación se nos abre" (p. 613). La línea narrativa que propone la investigación cualitativa, en tanto, ocupa unas técnicas que permiten el acceso a la subjetividad a través del lenguaje, junto con su análisis mediante el método hermenéutico caracterizado por la comprensión e interpretación del mundo simbólico y cultural que los individuos plasman en sus acciones, nos permite esclarecer, mediante el relato protagónico, cómo los individuos/as se relacionan con su entorno; viven o experimentan cambios en sus hábitos y costumbres; las transformaciones que generan para adaptarse a nuevos contextos. En suma, cómo se relacionan y cómo sus relaciones dan 
cuenta de una constante reelaboración de identidad propia en relación con el contexto institucional.

Como instrumento de recolección de información se utiliza la entrevista en profundidad semiestructurada. Con la elección de esta técnica se pretende obtener un relato "experienciado", en el que se da cuenta de las prácticas pasadas y presentes que recrean las entrevistadas en el contexto militar. Se prioriza esta técnica frente a otras como la observación en tanto este instrumento es más idóneo para captar los discursos.

Es importante mencionar que decidimos abordar a las entrevistadas mediante la organización. Esta nos solicitó tener claro el número de entrevistas y su duración con el fin de coordinar los permisos, por lo cual se define un número de ocho entrevistas de una hora de duración, dos por cada nivel de estudio de primero a cuarto año.

Algunas de las consultas realizadas son: ¿de qué manera se fue gestando el deseo de ingresar a la Escuela Militar? ¿Cómo describiría el ambiente dentro de la Escuela? ¿Considera que existen diferencias (de trato, estímulos, acceso, en las especializaciones, promociones, actividades de entrenamiento, actividades académicas) a nivel organizacional, o de la escuela entre hombres y mujeres? ¿Piensa que la organización ha logrado crear un ambiente que efectivamente incorpore en igualdad de condiciones a las mujeres? ¿Cuál es la misión o el rol de la mujer en las fuerzas armadas?

Por otra parte, aun cuando existen tres compañías, y en todas ellas participan mujeres, por decisión de la organización, todas las entrevistadas pertenecen a la misma unidad ( $1^{a}$ Compañía). Pese a que ello nos planteó entrar al campo con una muestra "ciega", en el sentido de que no se conocían mayores características de las entrevistadas, igualmente se contó con criterios de diferenciación relativos, principalmente, a la procedencia familiar y territorial. Así es como tres entrevistadas son parte de familias donde al menos un integrante es militar, y entre ellas dos son hijas de suboficiales. Mientras que cuatro de las entrevistadas provienen de regiones distintas a la metropolitana. 


\section{Aclaraciones teóricas}

La(s) identidad(es) es una temática de interés en la mayoría de las disciplinas sociales, por lo cual ofrece un desarrollo bibliográfico abrumador y a momentos desorientador. Frente a ello, optamos trabajar solamente con las propuestas planteadas desde la sociología y, en específico, los enfoques más contemporáneos.

Al respecto una de las propuestas más extendidas, hoy en día, para el análisis de las identidades, expone la caída del orden institucional, apuntando que a raíz de la globalización ya no hay posibilidad de mantener sistemas integrados de creencias. Este proceso llamado desinstitucionalización, apunta sobre la pérdida de vigencia de las instituciones para dar a los individuos los elementos para pensar su vida. De esta forma, los individuos quedan en una situación donde les corresponde, a ellos mismos, manejar las diversas situaciones, y están por tanto, constantemente eligiendo su destino, su trayectoria, un estilo de vida. Los autores que defienden esta tesis verán que estos procesos configuran una sociedad progresivamente inestable, imprevisible y riesgosa para los individuos (Giddens, 1997; Beck, 2001).

Otra de las propuestas para el análisis de las identidades, la encontramos en Castells (2003). Bajo la idea de una nueva sociedad, la sociedad red, el autor vislumbra la posibilidad de desafiar la supremacía del individuo, a partir de la idea de sujeto-actor. En este sentido las identidades no están a cargo exclusivamente del individuo, sino más bien los sujetos las construyen mediante sus identificaciones a colectivos con los cuales comparten símbolos, representaciones sociales y una orientación, lo que posibilita su acción colectiva.

Una tercera propuesta plantea que la identidad es la articulación de una historia personal y una tradición sociocultural, y que ambos registros no dejan de moverse históricamente (Dubet, 2002; Dubar, 2002; Martucelli, 2007). Este enfoque, en contraste con los previos, plantea, por una parte, que las identidades a pesar de estar más expuestas a una apertura cultural y a la multiplicación de los grupos 
de referencia, no pueden ser tan inestables como se quiere en el primer enfoque, en tanto no se pueden pasar por alto los roles, las presiones situacionales y las definiciones impuestas y heredadas de la realidad social. Y por otra, y por la misma apertura y multiplicación de referentes, tampoco pueden reducirse o ser sacadas directamente de las relaciones con una clase, comunidad, sexo, generación, como se plantea en el segundo enfoque. En tanto se reconoce que la identidad es una combinación de propiedades comunes a un grupo de actores, pero que también debe referirse a las propiedades individuales que se dan dentro de esas combinaciones, y que en su construcción median las instituciones y los roles, la perspectiva permite dar cuenta sobre la dialéctica entre lo social y lo individual y los elementos intermedios que median su relación.

Ya expuesto el enfoque teórico para reflexionar sobre la temática de la(s) identidad(es), abordaremos las otras consideraciones que sostienen nuestra pregunta de investigación, y que nos sirvieron para enmarcar los materiales que consideramos relevantes para la constitución de la(s) identidad(es) en el ámbito educativo militar de primer nivel.

La primera de ellas corresponde al género, ello no solo porque nuestra investigación instala a las mujeres y la construcción de su identidad en el centro, y este concepto posee un gran potencial para definir nuestra identidad. También, en la medida en que la perspectiva de género nos permite entender la organización militar como una cristalización de normativas provenientes de un sistema recreado bajo la lógica de la complementariedad genérica. En este sentido, comprendemos el género como un ordenador social, desde un punto de vista relacional y contextual, y como un sistema complejo con naturaleza recursiva que estructura normas, representaciones sociales, prácticas e identidades colectivas y subjetivas, al mismo tiempo que es estructurado por ellas. Y que, por otro lado, también sirve como marco interpretativo de la distribución del poder entre los grupos sociales (Scott, 1996; Butler, 2001).

Por otra parte, la revisión teórica realizada sobre esta temática se extiende para enmarcar el problema referido al tratamiento de la 
política de género inspirada en la igualdad de oportunidades, donde precisamente los estudios feministas y de género han elaborado definiciones y planteamientos teóricos para los conceptos de igualdad y diferencia. Particularmente interesante es lo que plantea Marques-Pereira (2004), quien indica que cuando estos conceptos son leídos como par opuesto, excluyen o consideran lo diferente como equivalente para incluir, lo cual articula la idea de igualdad formal. Pero que, sin embargo, histórica y sociológicamente, esta es una falsa oposición, en tanto, la igualdad se opone al privilegio y a la discriminación; y la diferencia a la similitud. Siguiendo estas claves teóricas, adoptamos el concepto de igualdad de género acuñado por PNUD "Igualdad no significa que las mujeres y los hombres lleguen a ser la misma cosa, sino que sus derechos, responsabilidades y oportunidades no dependan de si acaso ellos han nacido con sexo masculino o femenino. La igualdad de género implica que los intereses, necesidades y prioridades de mujeres y hombres son tomados en cuenta, reconociendo la diversidad de los diferentes grupos de mujeres y hombres" (PNUD, 2010; p. 29).

La segunda consideración atiende a la organización militar. La revisión teórica, en este caso, intenta articular desde un análisis sociológico un conjunto de elementos constituyentes de su ordenamiento -coordenadas valóricas, cultura organizacional, régimen de interacción jerárquico, sociabilidad (asociación) de tipo comunitario, y el concepto de vocación profesional- describiendo, con ello, la práctica institucional del sistema, y la ocupación/profesión como los otros materiales identificatorios significativos que se introducen en el proceso de formación de oficiales. Así también, recogimos la propuesta teórica de Goffman (2001) sobre las instituciones totales. Desde este conocimiento se plantean algunas premisas para leer la construcción de identidad en organizaciones con marcado carácter institucional, particularmente atendiendo a las fuerzas constrictivas que poseen las prácticas, que ahí se recrean, para promover un tipo de individuo. 


\section{Condiciones socioculturales activadas en la elección de la carrera:}

\section{La aproximación a la carrera militar}

Las condiciones socioculturales que se activan en la elección de la carrera militar varían entre aquellas cadetes que pertenecen a familias militares o bien poseen una vinculación directa y estable, y aquellas cadetes que no observan vinculación anterior con la organización.

En el primer grupo, el condicionante es de tipo institucional (Dubar, 2002), siendo las relaciones de parentesco con impronta tradicional lo que articula y conecta a la cadete con la organización "... yo veo a un militar y es como, chuta, mi familia pertenece a eso, mi familia es parte de eso, del Ejército” (cadete $1^{\circ}(2)$ ). Su elección aparece evidentemente dependiente de la herencia profesional del padre "...siempre dije que quería ser militar, que quería ser como mi papá" (cadete $1^{\circ}(2)$ ). Junto a ello, la exposición continua a relaciones que ponen en el centro la comunidad y actividad militar, se va traduciendo en un estar desde siempre insertas en el circuito, proporcionando un contexto que da la legitimidad necesaria para continuar estudios superiores en el Ejército, presentándose esta elección como una conclusión lógica del camino que han recorrido y cimentado durante toda su vida “... yo desde chica vivía, o sea siempre venía a la escuela, siempre desde chica he tenido a la Escuela en la mente. Entonces desde chica siempre quise ser militar, no tenía otro ámbito. (...) Y nunca tuve un ambiente que no fuera militar. Siempre viví, hasta el día de hoy vivo en villas militares, tengo amigos militares, iba a un colegio donde iban militares. Entonces toda mi vida ha girado en torno a militares" (cadete $2^{\circ}(1)$ ).

En este grupo de cadetes el impacto de los cambios en las relaciones de género es visible y se concreta adquiriendo un significado "rupturista" en lo biográfico-familiar (Beck-Gernsheim, 2003). Actualmente las hijas de militares pueden proyectarse estudiando el oficio/profesión de su padre, abriéndose la posibilidad de que sean ellas las que se hagan cargo del "legado familiar", cuestión que antes por tradición recaía normativamente en los hijos varones, quedando 
las mujeres al margen de esta posibilidad “... mi familia somos puras mujeres, hijas, somos tres, y yo soy la del medio y él decía (papá): pucha, que jamás se imaginó que una hija fuera a ser como él" (cadete $\left.4^{\mathrm{o}}(1)\right)$.

Por su parte, en aquellas cadetes que no poseen relación anterior con "el mundo militar" los condicionantes socioculturales que se activan son de tipo discursivo. En sus relatos circula una sensación de inestabilidad social que se traduce en inseguridad sicológica e inseguridad laboral (Giddens, 1997; Beck, 2001). Lo que configura un escenario propicio para que las cadetes vean en la educación militar, por su clima regulado, un lugar para acotar la incertidumbre que muestran otras alternativas académicas “...Me gusta que hay un respeto, no sé yo veo, de repente pienso a lo mejor si no hubiese entrado acá me hubiese ido a la universidad y estaría perdida totalmente... No, yo decidí centrarme y vivir esto. Entonces por ese lado yo digo: claro por eso elegí la formación militar" (cadete $3^{\circ}(1)$.

En este grupo prima una manera activa y gestionada de adherir a un colectivo, en la medida en que muestra un lazo más racional, provisorio e interesado, para realizar su proyecto profesional “... era lo que más me llamaba la atención, donde más me iba a desarrollar con lo que me gusta. Porque la malla abarca como varias cosas, aprendo lo militar, también ramos universitarios, que tengo la opción de hacer deporte, y los periodos de instrucción y de campaña, todas esas cosas. Era como bastante completo, como que era un paquete de cosas que me gustaban" (cadete $2^{\circ}(1)$ ).

Este hallazgo es susceptible de ser conectado con la apertura de la educación militar al mercado educativo terciario, definida cada vez más como equivalente a una carrera universitaria en cuanto a la incorporación de disciplinas/materias universitarias a la malla de estudio, como a la publicidad masiva en la promoción de la carrera. Lo relevante de esta relación es que manifiesta por un lado, que cada vez es menos necesario -para los postulantes- presentar credenciales del tipo de pertenecer a una familia militar, con lo cual el carácter heredable de la profesión se relativiza, y por otro, el que las mujeres 
puedan verse ahí, en tanto lugar de educación y no solo de formación de un sujeto masculino.

\section{Marcadores de identidad de la formación militar:}

\section{a) Los procedimientos}

En cuanto a los marcadores de identidad de la educación militar, analizamos a partir del discurso de las cadetes el proceso de regimentación, experimentado con mayor intensidad e incomodidad, en los primeros meses de carrera, reconociendo en él dos procedimientos que afectan a quienes ingresan a la escuela.

El primer procedimiento al cual llamamos despojos, reúne un conjunto de prácticas que afectan censurando y aminorando la capacidad de autonomía y discrecionalidad. Desinstalan las concepciones y prácticas precedentes en los planos del tiempo "...me habían explicado más o menos el régimen interno, que se habla, me decían: no, si uno igual tiene tiempo para estudiar, para descansar, pero la verdad estando dentro es totalmente diferente, no sé las horas libres, uno está como todo el día encerrada" (cadete $2^{\circ}$ (2)). En las relaciones y grupos de pertenencia "...La familia, porque era como 'pucha, hija, te echamos de menos, ven para acá'... y era como: no papá, sabes que no puedo, tengo que estar aquí, porque ya entré, que tengo que hacer tal cosa en la Escuela" (cadete $4^{\circ}(2)$ ). El espacio social e íntimo "...son cosas súper sencillas las que uno pasa, de dormir sola en la pieza, a que ahora duermo con tres compañeras a un lado, tres más, cinco en frente. De que es un baño para todas, uno antes ocupaba el baño y su familia, esas cosas son como fuertes" (cadete $2^{\circ}(2)$ ). Y la decisión autónoma "El año que se me hizo más difícil a mí, fue el primer año, y me dieron ganas de abandonar la escuela, quizás por no tolerar que alguien me mandara que fuera un año mayor que yo" (cadete $4^{\circ}(1)$ ).

Estas prácticas despojantes que experimentan las(os) alumnas(os) apuntan a reducir las múltiples formas identificatorias -sociales y culturales- a la sola influencia de la organización institucional militar. La intensa reglamentación de la "vida militar" 
separa y desinstala para hacer del mundo civil una desapropiación, y succiona para hacer que los alumnas/os cadetes no se sustraigan a la tendencia absorbente de la institución (Goffman, 2001). Es por ello que las otras esferas de la vida: relaciones sociales, familia, amistades, intimidad, ocio, se vuelvan completamente transables. Así es como crean y marcan una identidad social basándose en la diferencia entre dos mundos contrapuestos: civiles y militares "entonces ya la distancia, ya es mucha, ya no hay mucho feeling, pero siempre está el cariño (...) yo no me sentiría cómoda con mis amigas, las que están en la universidad, porque ellas tienen sus temas, tienen su espacio, tienen su grupito. Entonces yo como que no encajaría mucho hablando cosas como qué paso en la lectura de la orden, que me aporrearon por esto, que viste, no sé, que mi teniente, que mi cadete (...) como que, si, me siento limitada con ellas, porque no puedo expresarme" (cadete $2^{\circ}(1)$ ).

El segundo procedimiento que llamamos unificación, reúne un conjunto de prácticas que promueven e instalan concepciones colectivas que afectan principalmente el plano de la responsabilidad. Mediante sanciones “...si hay que sancionar a alguien, nos sancionan igual, sin observar quien es, simplemente se observa la falta. Y eso yo lo encuentro que es muy justo" (Cadete $4^{\circ}(1)$ ), relaciones de fraternidad "la única que tiene al lado es a la compañera que duerme al lado, la que forma al lado. Entonces las amistades ya llegan a ser como las hermanas" (cadete $2^{\circ}(2)$ ), y una concepción del trabajo que pone en el centro la idea de cuerpo "...y el trabajo en equipo, el de que todos tiramos para arriba, o sino todos nos caemos y nos volvemos a subir y que todos transpiramos la misma gota, eso es lo entretenido de la Escuela, en la educación, de que es todo por un bien común, no para trabajar por uno solo" (cadete $2^{\circ}(1)$ ) van componiendo en las cadetes un modo de acción colectivo, que apunta a la creación de compromiso y lealtad institucional.

Los procedimientos de unificación marcan la identidad mediante la pertenencia. De algún modo, ello trata sobre "colonizar" al individuo, convertirle en sujeto institucional (Zuliaka, 1989 en Molina, 1996), un sujeto que privilegie la noción de un "nosotros" sobre la noción de un "yo" "Nosotros los militares somos una familia, 
somos todos unidos y tenemos que trabajar juntos, para que todo salga bien" (cadete $1^{\circ}(1)$ ).

\section{b) El sujeto militar}

Otro de los marcadores de identidad de la educación militar, son las coordenadas simbólicas que caracterizan a la masculinidad en una versión tradicional. Las cuales se van incorporando en la subjetividad de quienes participan del proceso para la configuración de cuerpos fuertes, resistentes y capaces de dominarse y dominar.

$\mathrm{Al}$ respecto, los relatos muestran que convertirse en militar, exige del control y represión de las emociones que reflejen temor o debilidad "Entonces es como que tiene que aprender a controlarse, lo mismo de andar llorando, y todo eso" (cadete $3^{\circ}(1)$ ). La expresión de emociones y sentimientos, asociados al desaliento, producen descrédito y son interpretadas como desestabilizadoras de la imagen de autoridad y de la capacidad de ejercicio "O sea, claro a lo mejor lo único que quiero es irme para mi casa luego, pero no puedo bajar a formar con mis cadetes con la cara larga, porque ellos a uno lo tienen de ejemplo, o sea, cómo uno le va a decir a él, a ellos: oiga, motívese, sea enérgica...si uno está mal" (cadete $3^{\circ}(1)$ ). Por el contrario, el personaje militar que se va componiendo en torno a la exacerbación de emociones capaces de ser transmutadas en autoridad, actividad, energía y fuerza (Héritier, 1991; Olavarría, 2000), particularmente aquellas que presenten signos de agresividad como la rabia y el enojo "En la energía, se canaliza con la energía. Un recluta siempre tiene que estar "de los nervios", como dicen, siempre tiene que estar enérgico. Y toda esa rabia se canaliza en energía, corriendo más rápido, no sé, pegándole al fusil bien fuerte, todo eso" (cadete $1^{\circ}(1)$ ).

Por otra parte, el proceso de formación militar-despersonalizado, indiferenciado y homogeneizante- se inspira en un sujeto normativo abstracto que estaría por encima de los sujetos reales, sean estos hombres o mujeres. En estos términos, se produce una aparente neutralización, en tanto borra el juego entre identidades diferenciales femenino/masculino e instaura en su lugar una identidad única y totalitaria, la militar “... acá no va a haber los oficiales femeninas y los 
masculinos, vamos a ser todos oficiales de Ejército" (cadete $3^{\circ}(1)$ ). El uso y aplicación de una definición universal y despersonalizada, ha posibilitado presentar como neutros los estándares, normas y valores necesarios para calificarse como oficiales. Las cadetes aparecen como individuos abstraídos de la naturaleza respondiendo a normas y patrones universales, que por la aparente neutralidad que comportan funcionan como indicadores de igualdad “...tenemos que tener las mismas exigencias, los mismos parámetros (...) para que una misma después no ande exigiendo. Porque las mujeres exigen igualdad también" (cadete $3^{\circ}(1)$ ).

Lo expuesto problematiza el que las mujeres puedan ser representantes de este universal. Y ello se refuerza en la medida en que la abstracción normativa y universalidad son empleadas como las garantías de igualdad para los partícipes reales en este espacio. De alguna manera, que la universalidad sea la garante de igualdad para los diferentes, confirma la exclusión de quienes no se conformen a las normas dominantes (Marques-Pereira, 2004).

\section{El tratamiento de las nociones de igualdad y diferencia en la experiencia cotidiana en la Escuela y su traspaso al ejercicio laboral}

Cotidianamente para las cadetes la igualdad se traduce en recibir los mismos tratos y exigencias "...en cuanto a régimen interno, como ejercicios de escuela o campañas de cursos, en las secciones que hacemos todos los días, ninguna diferencia, somos todos iguales, el trato es igual" (cadete $4^{\circ}(2)$ ). La concepción de la igualdad formulada en relación con el trato, de algún modo, lo que hace es igualar desplazando las diferencias entre hombres y mujeres, bajo el criterio de ley general válida para todos "...tenemos que tener las mismas exigencias, los mismos parámetros, y por eso hay cosas que tienen que ser iguales para ambos" (cadete $3^{\circ}(1)$ ).

Una de las consecuencias que tiene este tratamiento de la noción de igualdad basada en el trato, es que suspende las diferencias creando la fantasía de que hombres y mujeres están "a la par" y poseen los mismos deberes y derechos. Otra es que a la negativa a admitir el 
registro de la diferencia en la construcción de un discurso a favor de la igualdad de géneros endosa a las cadetes la obligación de sostener la igualdad, y cualquier reclamo de ella. “.... siempre dicen 'no quieren igualdad, ya poh entonces se aguantarán' (...) Y en realidad yo creo que eso está bien, porque hay gente que abusa de eso (...) Yo no siento que las mujeres en ningún caso pidan regalías de nada. Por lo mismo, porque acá siempre nos dicen 'bueno no buscan igualdad"' (cadete $3^{\circ}(1)$ ). Así también, esta concepción, promueve que algunas de las cadetes transgredan su diferencia -en tanto diferencia negativaajustándose a la norma masculina, para ser reconocidas como pares y validadas como iguales "es como 'ya las mujeres tienen que hacer barra', ya, por último me voy a colgar de la barra, pero algo voy a hacer. No me voy a quedar callada, ni me voy a quedar atrás" (cadete $4^{\circ}(2)$ ). O bien la sufran atribuyéndole un significado inferior asumiendo una relación jerárquica de subordinación respecto a los cuerpos de los varones "...las mujeres no estamos como ciento por ciento implementadas. Porque, por ejemplo, la vida de milicia, la vida militar, la mujer en combate no funciona" (cadete $2^{\circ}(1)$ ).

Por otra parte, este discurso a favor de la igualdad de género que sigue el criterio de ley general válida para todos, pone en circulación una lógica de los méritos individuales y privados que responsabiliza a los individuos de hacerse cargo de su trayectoria. "A mí en primero me dijeron, ustedes parten de una misma línea, ustedes después se hacen el lugar, porque todos tenemos distintas capacidades para estudiar, todos tenemos distintas capacidades para hacer deportes, todos hacen sus competencias (...) Acá uno hace su arma y su servicio. Depende de cada uno" (cadete $3^{\circ}(2)$ ). Sin embargo, la lógica entra en contradicción cuando se trata de mujeres. Al respecto el análisis muestra que las cadetes son abordadas de modo indiferenciado, como parte de la categoría mujer, para efectos de distribución de oportunidades de acceso, trabajo y diseño de la trayectoria laboral. Es decir, sus méritos individuales no son reconocidos a pesar de que en sus experiencias cotidianas y en el proceso de formación general, hacen y reciben el mismo trato que los hombres.

Lo expuesto hasta ahora, nos permite afirmar que el tratamiento de la igualdad de género al interior de la Escuela, al parecer solo 
apunta sobre la igualdad de trato, dejando fuera la igualdad de derechos y ejercicio, lo cual se pone de manifiesto mediante los bienes de calificación a los que pueden acceder. Al respecto, los antecedentes revisados confirman que para las mujeres existen lugares vetados en cuanto a la carrera profesional, encontrando limitadas sus expectativas a dos de las tres áreas de especializaciones profesionales: Armas de apoyo al combate y Servicios e Intendencia, e incluso existe exclusivamente para ellas Servicio Femenino (ver tabla n. ${ }^{\circ} 1$ ). A partir de estas variantes profesionales, las cadetes deben ajustar sus expectativas a posiciones socialmente sexuadas, encontrando sus lugares de actividad preferentemente en las áreas administrativas.

Tabla n. $^{\circ}$ 1. Especializaciones optadas por cadetes entre los años 1996 y 2009

\begin{tabular}{|l|c|c|c|c|c|c|c|c|c|c|c|c|c|c|}
\hline Especialización & 96 & 97 & 98 & 99 & 00 & 01 & 02 & 03 & 04 & 05 & 06 & 07 & 08 & 09 \\
\hline RR.PP. y personal & 9 & 9 & & & & & & & & & & & & \\
\hline Finanzas e informática & 7 & 6 & & & & & & & & & & & & \\
\hline Material de guerra & & & 3 & 4 & 5 & 6 & 2 & 1 & 5 & 2 & 2 & 7 & 6 & 6 \\
\hline Intendencia & & & 6 & 7 & 5 & 6 & 3 & 1 & 5 & 1 & 3 & 5 & 8 & 8 \\
\hline Servicio femenino & & & 5 & 5 & 6 & 8 & 3 & 4 & 5 & 6 & 7 & 7 & 5 & 7 \\
\hline Artillería & & & & & & & 4 & 1 & 5 & 3 & 3 & 4 & 2 & 2 \\
\hline Ingenieros & & & & & & & 3 & 1 & 5 & 3 & 2 & 4 & X & X \\
\hline Telecomunicaciones & & & & & & & 2 & 1 & 5 & 2 & 2 & 7 & 4 & 4 \\
\hline Total & 16 & 15 & 14 & 16 & 16 & 20 & 17 & 9 & 31 & 18 & 19 & 31 & 25 & 27 \\
\hline
\end{tabular}

Fuente: documentos de trabajo, Escuela Militar de Chile.

Hasta ahora hemos expuesto que la construcción de la identidad profesional a partir de las especializaciones profesionales, al interior de la Escuela, es diferente entre hombres y mujeres por concretarse en prioridades y esferas de acción distintas. Esta situación es una constante no exclusiva de las cadetes, pues se puede observar ampliamente en todos los ámbitos productivos en que actúan y se encuentran presentes las mujeres. Sin embargo, cada ámbito recrea mecanismos específicos para mantener la división sexual del trabajo y las convenciones sociales. En específico, en el Ejército y para las actividades de combate directo, el análisis da cuenta de dos mecanismos que posibilitan seguir perpetuándolas, además de promover abiertamente la exclusión. El primero de ellos que hemos llamado naturalización del mito, respecto de lo discursivo y representacional alude a simbolizar doblemente a las mujeres 
(Lagarde, 1992). De alguna manera, se trata de vitalizar como sustento de exclusión, aquellos mitos que representan a las mujeres como peligrosas por un lado, y frágiles por otro: hay que desplazarlas en tanto son una amenaza para el grupo "Hay cosas que me han explicado, también me llaman la atención... por qué, por ejemplo, la mujer no puede ser infantería... porque al hombre le choca ver a una mujer muerta... eso lo entiendo, antes no lo entendía, ahora lo entiendo" (cadete $1^{\circ}(2)$ ), y hay que desplazarlas para protegerlas, frágiles susceptibles de ser maltratadas en el campo de batalla “... por ejemplo, ya va un batallón y hay tres mujeres, y son como 'las cachorritas', son como 'los pollitos', y todos las cuidan, entonces si una se muere en combate, todos van a tener la moral baja, y no van a combatir bien, entonces puede ser por eso" (cadete $1^{\circ}(1)$ ).

El segundo mecanismo que hemos llamado negación de las individualidades, actúa en cuanto a lo relacional. Las mujeres son remitidas a una versión universal y esencialista de la mujer. Por un lado sus diferencias y diversidad, en términos de potencialidades físicas por ejemplo, no son reconocidas, ninguna mujer puede presentarse a rendir las pruebas en dichas áreas "... a veces como la pregunta por qué la mujer no puede ser infante, y es como 'no, porque no les da la condición física' (...) “... es como cerrado la... 'no para las mujeres' (...) Eso me parece mal. Porque yo creo que independientemente de hombre o mujer, debería verse si alcanza las cualidades para... antes de cerrarse" (cadete $2^{\circ}(2)$ ). Y por otro, son cuerpos -indiferenciadosremitidos a los papeles sociales de género, intercambiables como madres, esposas, hijas “...Ellos se acuerdan de la mamá, la polola, de las hijas, entonces se ponen a llorar, (...) se ponen tristes, y no pueden seguir luchando, está comprobado" (cadete $3^{\circ}(2)$ ).

\section{Referentes de género que se relevan en la construcción de la identidad femenina}

El último objetivo específico del estudio, tuvo la intención de dar luz sobre los referentes de género, ello en el marco del debate contemporáneo que ha puesto de manifiesto la emergencia de "nuevas feminidades" que se alejan de los referentes más tradicionales, y de una definición rígida de lo femenino y masculino. 
El análisis de los relatos permite reconocer que en la educación militar, los referentes tradicionales marcan presencia y la distinción entre lo femenino y lo masculino parece seguir vitalizando un ordenamiento binario y poco flexible. En este sentido, el modelo de identidad femenina al interior de la organización militar se relaciona estrechamente con aquella versión que -para producirla- excluye lo masculino. Adhiriendo a la división sexual del trabajo para acordar tareas diferentes entre hombres y mujeres, y a la esencialización de los significados que fundan lo masculino y femenino, para acordar, a su vez, normas y modos de expresión distintas para construir los cuerpos (Butler, 2001; Bourdieu, 2005).

Especialmente en lo que se refiere al ejercicio -actividades, funciones y trabajos-, las cadetes responden favorablemente a las identificaciones grabadas en la división sexual del trabajo. Asimilan como propia la reproducción de la vida cotidiana estableciendo una fuerte identificación del trabajo que desempeñarán como oficiales del Ejército con lo doméstico "...yo siempre he dicho que la mujer tiene el rol de calcular todo, es como la dueña de casa, quizás se preocupa de muchas cosas, se preocupa de la comida de los hijos, se preocupa que no falte esto, lo otro, uno administra, uno como mujer tiene la cualidad de administrar muchas cosas y saber dónde faltan determinadas cosas. Y más que nada ese rol o esa misión es la que tiene la mujer, que es de administrar los recursos" (cadete $\left.4^{\circ}(1)\right)$.

La maternidad, dimensión tradicional de la feminidad, a pesar de que aún no son madres, define con bastante independencia otros ejes alternativos de identificación, en la medida en que se prefieren actividades que les permitan, en el futuro, experimentarle más aliviadamente "...también uno no quiere, por ejemplo, yo quiero tener familia, entonces eso igual me limita mucho a elegir un arma, porque no alcanza el tiempo, entonces igual uno tiene que compensar o planificarse de aquí a futuro" (cadete $2^{\circ}(1)$ ). Ello pone en evidencia que los costos de llevar una vida militar se hacen más críticos para las mujeres, e implican arreglos, postergaciones o renuncias que el caso de los varones, debido a la vigencia de la división socio-sexual de géneros complementarios, podrían no ser tan relevantes, y permitirles 
sostener, con mayor soltura, la profesión como eje identificatorio "Pero yo creo que para la mujer de armas también ha sido difícil, por las experiencias que he escuchado, que se tiene que ir a campaña y la guagüita está chica" (cadete $3^{\circ}(1)$ ).

La educación militar, de trato indiferenciado, tensiona y disloca la identidad tradicionalmente entendida como "femenina", en cuanto a las normas y modos de expresión, llamándoles a suprimir aquellas expresiones acordadas socialmente como "de mujeres". El conflicto es visible en la necesidad de negar parte de lo "femenino". Parecen entonces, obligadas a masculinizarse en términos de volverse fuertes, mantener control y dominio para insertarse y convertirse en militares. Es por ello que, en el caso de algunas entrevistadas, la presión de asimilar la formación es significada como una exigencia de igualación con los hombres mediante su masculinización “...a veces nos tratan como hombres, que no ven la consecuencia que pudiera llegar a tener (...) No sé, por lo menos a nosotras nos falta como la parte de más feminidad porque uno no es que la pierda, sino que la pospone. Como que eso más. Y obviamente como que a los hombres no les gusta que una ande como saltando, gritando, como que no va con la vida militar" (cadete $2^{\circ}(1)$ ). En este escenario, las cadetes, para convertirse en militares, tienen que lidiar con la distancia que se establece entre aquellos significados tradicionalmente masculinos, sus cuerpos de mujer y la construcción socio-biográfica de la identidad femenina.

La confusión en la definición de sí mismas en tanto género que se expresa en la inquietud de ser capaces de mantener una concepción de feminidad, las empuja a retomar lo conocido, el modelo tradicional de género, al cual recurren como referente de identidad (Sharim, 2005). Así, las cadetes entran a este mundo público y de predominio masculino en clave femenina. Buscando una suerte de "refugio" en los modelos tradicionales, trasladan actitudes y lenguajes, formas de trato y relación, que en la mayoría de las ocasiones son ajenas porque, al parecer, pertenecen a otro sitio "Como que con las mujeres entro como la parte emocional como de la tropa. Yo creo que están más preocupadas de lo que le pasa al que está a cargo de uno, que solo instruirlo, forme ahí, marche ahí" (cadete $2^{\circ}(2)$ ). Inspiradas en 
la figura maternal administradora del afecto, preocupada del otro e intuitiva, muestran una forma distintiva en el ejercicio del mando, en contraposición al ejercido por los varones, quienes, según las cadetes, no cuentan con estas habilidades. "En eso me di cuenta, de que los hombres no, mi comandante de escuadra era como... no se dan cuenta de cosas, si estás bien, sino es como que ven siempre lo mismo, la mujer no, como que sabe al tiro cuando uno anda como achacada, o anda bajá (...) no sé, yo me he fijado, yo tuve muchos mandos mujer durante primer año, son más detallistas, y son como más preocupadas. Los hombres son como más desarmados, como que no se fijan a veces si estás bien o no" (Cadete $2^{\circ}(2)$ ). Este liderazgo distintivo de las mujeres responde a la aplicación de un saber de género adquirido tempranamente por las cadetes, en tanto mujeres/ madres y bajo este saber no solo comprenden su ejercicio, sino también extraen materiales de identificación (Lagarde, 1992).

\section{Conclusiones}

Chile es el primer país en Latinoamérica en incorporar mujeres a la oficialidad, y actualmente, junto a Argentina, sustenta y legitima su integración en un marco legal que recoge la perspectiva de derechos humanos y de la mujer. En este sentido, nuestro país se ubica a la vanguardia en temas de equidad de género en políticas públicas de defensa en el contexto sudamericano.

A consecuencia de la formulación de una política de participación de las mujeres en las fuerzas armadas (2005), las cadetes que cursan hoy la carrera de oficiales de Ejército en relación con generaciones más antiguas viven una experiencia distinta. En la actualidad hombres y mujeres conviven en las mismas instalaciones, comparten un plan de estudio común y las especializaciones a que pueden optar las mujeres se han ampliado. Sin negar la importancia que tiene este conjunto de reformas en la generación de un espacio para que las mujeres participen como parte de la fuerza en el Ejército, ello solo es uno de los aspectos para considerar si se está pensado en producir un movimiento sustantivo hacia la cualificación de las mujeres que participan de este proceso. 
Nuestra investigación revela que aún persisten obstáculos y tensiones, particularmente en el plano ocupacional e institucional que limitan una mayor igualdad de derechos y oportunidades. Ello queda en evidencia, especialmente cuando se toma en cuenta que su incorporación no ha resultado de un proceso de modernización interno de la organización, sino más bien de una iniciativa política. Y justamente en referencia a ello, es importante reconocer claramente qué esperan las organizaciones con su ingreso, con el fin de despejar, por un lado, la posible instrumentalización política que presenta el ingreso de mujeres como uno de los indicadores de modernización institucional y, por otro, una posible instrumentalización laboral que les asigne casi en exclusividad plazas administrativas.

En otro aspecto, desde una perspectiva cultural e ideológica, nuestra investigación evidencia que el ingreso de mujeres no es equivalente a un proceso de modernización institucional, que recoja justamente los cambios en las relaciones de género a nivel social, que, por lo demás, sustentan la incorporación de mujeres a las organizaciones militares en general y al Ejército en particular. Un proceso de modernización institucional en esa dirección tendría que realizar cambios profundos en las formas de representación simbólica que "distribuye" identidades de género esencializadas, roles de género que atienden a la división productivo/reproductiva, y muy especialmente en una socialización profesional marcada por símbolos y valores identificados como masculinos. Así también y especialmente en las exigencias propias de la vida militar, aquellas que involucran la vida privada de sus integrantes y que para las mujeres presentan mayores tensiones y conflictos, tomando en cuenta las determinaciones de género aún vigentes.

A partir de lo que hemos desarrollado a lo largo del texto, nos queda por decir que el análisis de la configuración de identidad de las mujeres cadetes en la educación militar, muestra que existe al menos una distancia entre los discursos generalizados que hacen aparecer su inclusión como un signo potente del desvanecimiento de las fronteras entre los sexos, y los discursos y prácticas al interior de la organización militar, en la medida en que en ellos se agudiza la división masculino/ femenino en conformidad al rol genérico y la división social del 
trabajo, lo que motiva la construcción de una identidad, para el caso de las mujeres, que refuerza el rol femenino tradicional, enfatizando las representaciones sociales, imágenes y estereotipos femeninos tradicionales, y por tanto promueve la aceptación naturalizada y sexualizada de una identidad por complemento.

Por último, para futuras investigaciones y a modo de contraparte, nos debemos preguntar, como ya lo decíamos, qué esperan las organizaciones con su ingreso y cuáles son los cambios que están dispuestas a realizar, en la medida en que el proyecto de que ingresen mujeres en igualdad de oportunidades, si se asume cabalmente, podría transformar la organización desde un tipo institucional a un tipo ocupacional.

\section{Referencias bibliográficas}

Beck, U. (2001) Vivir nuestra propia vida en un mundo desbocado: individuación, globalización y política. En: Giddens, A. y Hutton W. (eds.) En el límite. La vida en el capitalismo mundial. Barcelona: Tusquets Editores.

Beck-Gernsheim, E. (2003) De "vivir para los demás" a "vivir la propia vida". En: La individualización. Barcelona: Paidós.

Bourdieu, P. (2005) La dominación masculina. Barcelona: Anagrama.

Butler, J. (2001) El género en disputa: el feminismo y la subversión de la identidad. México: Paidós.

Castells, M. (2003) La era de la información. Vol. II: El poder de la identidad. México: Siglo Veintiuno.

Dubet, F. y Martuccelli, D. (1998) En la escuela, sociología de la experiencia. Buenos Aires: Losada.

Dubet, F. y Martuccelli, D. (1999) ¿En qué sociedad vivimos? Buenos Aires: Losada.

Dubar, C. (2002) La crisis de las identidades. La interpretación de una mutación. Barcelona: Bellaterra.

Echeverría, C. (1994) Apuntes sobre la integración de la mujer en las Fuerzas Armadas. En: Cuadernos de estrategia (68). España. pp. 67-74.

Giddens, A. (1997) Vivir en una sociedad postradicional. En: Modernización reflexiva. Madrid: Editorial Alianza Universidad, pp. 75-136.

Goffman, E. (2001) Internados. Buenos Aires: Amorrortu. 
Héritier, F. (1991) La sangre de los guerreros y la sangre de las mujeres. En: Alteridades. 1(2): p. 92-102.

Lagarde, M. (1992) Identidad femenina. En: Seminario Género, Políticas Públicas y Desarrollo. CEM, Unicef, Sernam. Santiago.

Lucero, M.R. (2009) Las mujeres en las fuerzas armadas argentinas. Breve análisis sobre su participación y estado actual de la situación. UNISCI Discussion Papers, n. ${ }^{\circ}$ mayo-sin mes, pp. 36-49.

Ministerio de Defensa Nacional de Chile (2005) Libro de Defensa Nacional: Separata de actualización 28: Participación de las mujeres en las Fuerzas Armadas. Chile: Ministerio de Defensa Nacional de Chile.

Martucelli, D. (2007) Gramáticas del individuo. Buenos Aires: Losada.

Martucelli, D. (2007) Cambio de rumbo: La sociedad a escala del individuo. Chile: LOM.

Marques-Pereira, B. (2004) Cuotas y paridad: miradas cruzadas entre Bélgica y Francia a la luz de la teoría política sobre ciudadanía y género. En: Igualdad de derechos. Igualdad de oportunidades. Igualdad de ejercicio. Chile: Editorial Instituto de la Mujer.

Molina, J.F. (1996) Quintas y servicio militar: Aspectos sociológicos y antropológicos de la conscripción (Lleída 1878-1960). Tesis doctoral Universitat de Lleída, España.

Papadópulos, J. y Radakovich, R. (2005) Educación superior y género en América Latina y el Caribe, Capítulo 8. En: Estudio Comparado de Educación Superior y Género en América Latina y el Caribe. IESALC.

Programa de Naciones Unidas para el Desarrollo (2010) Desarrollo Humano en Chile. Género: Los desafíos de la igualdad. Santiago: PNUD.

Olavarría, J. (2000) De la identidad a la política: Masculinidades y políticas públicas. Auge y ocaso de la familia nuclear patriarcal en el siglo XX. En: Olavarría, J. Masculinidad/es: identidad, sexualidad y familia: Primer encuentro de estudios de masculinidad. Santiago, Chile: Flacso.

Scott, W.J. (1996) El género: Una categoría útil para el análisis histórico. En: El género. La construcción cultural de la diferencia sexual. México: Editor Miguel Ángel Porrúa.

Sharim, D. (2005) Identidad y roles de género. Una aproximación desde los relatos de vida. Tesis doctoral en Sciences Psychologiques. Bélgica.

Vieytes, R. (2004) Metodología de la investigación de las organizaciones, mercado y sociedad. Epistemología y técnicas. Argentina: Editorial de las Ciencias. 
190 UNA APROXIMACIÓN A LA CONSTRUCCIÓN DE IDENTIDAD DE MUJERES CADETES EN LA ESCUELA MILITAR DEL EJÉRCITO DE CHILE - Claudia Pérez Bravo

Villalobos, P. (2007) Participación de las mujeres en las Fuerzas Armadas y de Orden. Políticas para promover la inserción de la mujer en la vida pública. Mesa de Trabajo Pro-Género. 2007. Disponible en: http://www.fes.cl/ documentos/Genero/progenero2007/villalobos.pdf

Recibido: 30/09/2011

Aceptado: 11/11/2011 


\section{Anexo n. ${ }^{\circ} 1$}

\section{Requisitos de postulación}

Los requisitos que se le exigen al postulante en la actualidad son:

- Ser chileno(a) de acuerdo a la Constitución Política de la República de Chile. Soltero(a), sin hijos y mantenerse en esta condición mientras permanezca como alumno en la Escuela Militar.

- Salud y condición física compatibles con las exigencias de la vida militar de acuerdo con la reglamentación vigente.

- Reunir las condiciones psicológicas para desarrollar una vida ligada al sistema militar que esté de acuerdo con los parámetros que exige la institución.

- No registrar ni haber registrado antecedentes penales. Esto es, no haber sido procesado ni condenado por delito alguno y mantenerse en esta condición mientras permanezca como alumno en la Escuela Militar.

- Estar cursando o haber cursado el último año de enseñanza media y acreditar su aprobación al momento de postular o terminarla.

- Edad máxima 22 años al 10 de febrero del año de ingreso.

- Estatura mínima de 1,70 y 1,65 m, para postulantes masculinos y femeninos, respectivamente.

- Ocupar una vacante de acuerdo al puntaje obtenido. 


\section{Anexo n. ${ }^{\circ} 2$}

\section{Línea de carrera de oficiales femeninas}

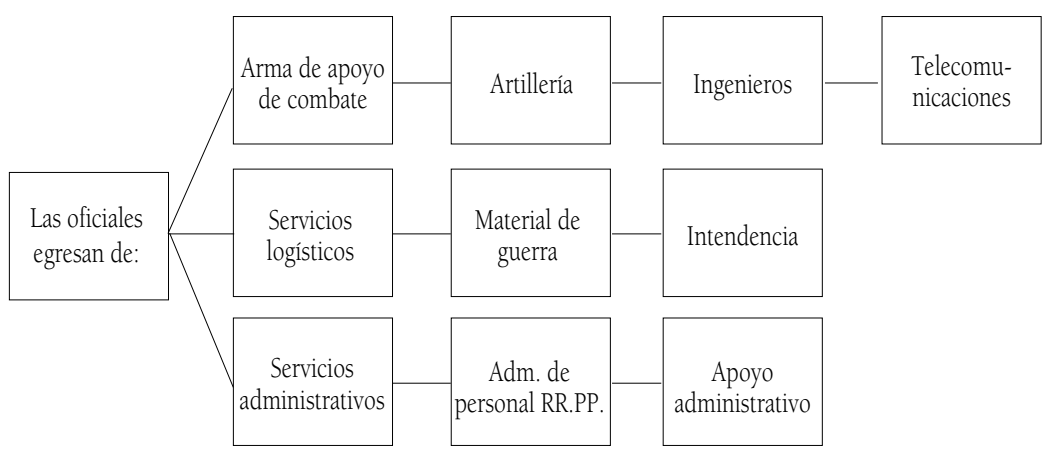

Elaborado por el Ejército de Chile. Se encuentra en línea en http://www.ejercito.cl/nuestra_fuerza/ mujer_ejercito.php 